\title{
ESTUDO IN VITRO DA HABILIDADE PREDATÓRIA DE Monacrosporium robustum SOBRE Heterodera glycines"
}

\author{
ARLETE S. MAIA ${ }^{* * *}$, JAIME M. DOS SANTOS² \& ANTÔNIO O. DI MAURO ${ }^{3}$ \\ 1,2 Departamento de Fitossanidade; ${ }^{3}$ Departamento de Fitotecnia, Universidade Estadual Paulista, Faculdade de Ciências \\ Agrárias e Veterinárias, Campus de Jaboticabal, CEP 14884-900, Jaboticabal, SP, e-mail: asmaia@fcav.unesp.br
}

(Aceito para publicação em 27/04/2001)

Autor para correspondência: Arlete Silveira Maia

MAIA, A.S., SANTOS, J.M. dos. \& DI MAURO, A.O. Estudo in vitro da habilidade predatória de Monacrosporium robustum sobre Heterodera glycines. Fitopatologia Brasileira 26:732-736. 2001.

\section{RESUMO}

O fungo nematófago Monacrosporium robustum foi detectado, isolado e identificado pela primeira vez de solos do Brasil, em maio de 1999, no Laboratório de Nematologia do Departamento de Fitossanidade da Universidade Estadual Paulista (UNESP), Campus de Jaboticabal, São Paulo, tendo sido o potencial como agente de biocontrole do nematóide de cisto da soja, Heterodera glycines raça 3 observado ao microscópio eletrônico de varredura (MEV) (Maia \& Santos, 1999). Na presente pesquisa, detalhes das estruturas de captura, tamanho, forma e septação dos conídios, bem como nematóides capturados pelo fungo foram documentados. Monacrosporium robustum produz micélio hialino, e as estruturas de captura são constituídas por ramificações adesivas, na forma de protuberâncias verticais que surgem das hifas, medindo, em média, $10 \mu \mathrm{m}$ de comprimento e 5 $\mu \mathrm{m}$ de diâmetro. Uma substância gelatinosa desprende- se dessas estruturas, ao contato com o nematóide, aprisionandoo. Os conídios do fungo são hialinos, fusóides com dois ou quatro septos, às vezes, cinco. Conídios jovens são asseptados e piriformes. Sob condições de laboratório, esse fungo predou $100 \%$ dos ovos e dos juvenis de segundo estádio de $H$. glycines e formas ativas de Panagrellus sp., no período de $72 \mathrm{~h}$ da exposição desses nematóides ao fungo.

Palavras-chave adicionais: controle biológico, fungo nematófago, nematóide de cisto da soja, microscopia eletrônica de varredura.

\section{ABSTRACT \\ In vitro study of the predatory ability of Monacrosporium robustum on Heterodera glycines}

The nematophagous fungus Monacrosporium robustum was detected, isolated and identified for the first time in Brazil, in May 1999, at the Departamento de Fitossanidade of the Universidade Estadual Paulista (UNESP), Câmpus of Jaboticabal, São Paulo, and its potential as a biological control agent of the soybean cyst nematode (Heterodera glycines) race 3 was evaluated with a scanning electron microscope (SEM). Details of the capture structures such as adhesive nets, size, septation and conidia shape, as well as nematodes captured were documented. Monacrosporium robustum produces hyaline mycelium and the capture structures are adhesive branches that rise vertically from the hyphae, averaging $10 \mu \mathrm{m}$ in height and $5 \mu \mathrm{m}$ in width. A gelatinous substance is freed from these structures when in contact with nematodes, capturing them. The conidia of the fungus are hyaline, fusiforme with two or four, and sometimes five, septa. The young conidia does not have septum. In laboratory, $100 \%$ of the eggs and second stage juveniles of $H$. glycines and the active forms of Panagrellus sp. were captured in $72 \mathrm{~h}$ of exposure of these nematodes to the fungus.

\section{INTRODUÇÃO}

A ocorrência de fungos predadores de nematóides, em diferentes ecossistemas, no Brasil, tem sido constatada por vários autores (Naves \& Campos, 1991; Santos et al., 1991; Ferraz et al., 1992; Maia \& Ferraz, 1993; Maia et al., 1993;

\footnotetext{
*Parte da tese de Doutorado do primeiro autor. Universidade Estadual Paulista, Faculdade de Ciências Agrárias e Veterinárias, Campus de Jaboticabal. **Bolsista da CAPES
}

Santos, 1996; Ribeiro et al., 1999b). A grande maioria dos fungos nematófagos isolados e/ou testados para o controle de Heterodera glycines Ichinohe, 1952 e Meloidogyne spp. é do tipo oportunista (Carneiro \& Gomes 1993; Chen et al., 1994; Chen et al., 1996; Coimbra et al., 1999; Freitas et al., 1999; Mizobutsi et al., 1999).

Santos (1991) e Dalla Pria (1992) observaram pronunciado efeito antagonista de Monacrosporium ellipsosporum (Grove) Cooke \& Dickinson sobre Meloidogyne incognita (Kofoid \& White, 1919) Chitwood, raça 3. Monacrosporium 
robustum McCulloch foi descrito por Janet S. McCulloch em 1977, isolado de amostras de solo em Queensland, na Austrália.

Em outro estudo, Santos (1996) avaliou o potencial in vitro de 18 isolados de fungos nematófagos a $H$. glycines, Pratylenchus brachyurus (Godfrey, 1929) Filipvej \& Schuurmans Stekhoven, 1936, Meloidogyne arenaria (Neal, 1889) Chitwood, 1949, M. javanica (Treub, 1885) Chitwood, 1949 e M. incognita raça 3. De acordo com a autora, Arthrobotrys robusta Duddington, M. ellipsosporum e outros três isolados não identificados, pertencentes à subdivisão Mastigomycotina, foram os mais eficientes quanto ao parasitismo e à predação dos fitonematóides testados, com exceção de $H$. glycines. No entanto, sob condições de casa de vegetação, em cultivos seqüenciais de feijoeiro (Phaseolus vulgaris L.), tomateiro (Lycopersicon esculentuum Mill.) e alface (Lactuca sativa L.), M. ellipsosporum foi o mais eficiente na redução da população das três espécies de Meloidogyne mencionadas.

A habilidade dos fungos nematófagos em colonizar a rizosfera tem sido apontada como uma característica importante de um agente do biocontrole. Persson \& Jansson (1999) estudaram, comparativamente, a colonização da rizosfera de tomateiro por 38 isolados de fungos nematófagos e avaliaram o controle de M. incognita e M. javanica por esses fungos, em casa de vegetação. Dentre os fungos, Arthrobotrys dactyloides Drechsler, A. superba Corda, $M$. ellipsosporum e M. gephyrophagum (Drechsler) Subramanian foram os mais freqüentes em rizosfera de tomateiro. Monacrosporium ellipsosporum e M. gephyrophagum, quando introduzidos no solo em alginato de sódio, exibiram grande capacidade de colonizar a rizosfera e esporularam duas vezes mais que os outros fungos em estudo. Entretanto, nenhum reduziu os danos causados pelos nematóides em plantas de tomateiro.

Ribeiro et al. (1999a) avaliaram a capacidade predatória de 59 isolados de Monacrosporium spp. frente a $M$. javanica e $H$. glycines. Esses autores observaram que a predação de juvenis de $M$. javanica variou de 71 a $100 \%$ para 27 isolados, enquanto, para $H$. glycines, 26 isolados não exerceram qualquer predação. Os outros 33 isolados exibiram máxima predação de apenas 1,2\% de juvenis desse nematóide. Todos os isolados predaram Panagrellus sp.

Maia \& Santos (1995, 1997 e 1999) ilustraram, ao MEV, as estruturas e formas de captura de Arthrobotrys brochopaga Drechsler, A. conoides Drechsler, A. musiformis Drechsler, A. oligospora Fresenius, Dactylella leptospora Drechsler, Harposporium sp.; M. cionopagum Drechsler, M. cystosporum Cooke \& Dickinson e M. ellipsosporum. Chen \& Dickson (1996) examinaram, através de microscopia óptica, MEV e microscopia eletrônica de transmissão (MET), a capacidade de penetração na parede de cistos de $H$. glycines por 12 espécies de fungos. $O$ tempo de penetração variou de um dia a um mês. Por ocasião da penetração, as hifas ficavam aderidas à parede dos cistos. Os autores encontraram evidências de que houve dissolução da parede, formando-se orifícios de penetração.

O presente trabalho teve como objetivo estudar, através da microscopia eletrônica de varredura, o modo de ação de M. robustum sobre ovos e juvenis de segundo estádio de $H$. glycines.

\section{MATERIAL E MÉTODOS}

O estudo foi conduzido no Laboratório de Nematologia do Departamento de Fitossanidade da UNESP/ FCAV, Campus de Jaboticabal, São Paulo. O fungo foi isolado de amostras de solo de seringais da região geoeconômica de Rondonópolis, MT. Para a detecção e isolamento do fungo, foi utilizado o método de espalhamento de solo, segundo Barron (1977), modificado por Santos (1991). Caracteres morfométricos do fungo foram obtidos nos microscópios óptico e eletrônico de varredura. Foram medidos o comprimento e a largura de 30 conídios ao microscópio óptico. Utilizou-se um sistema de aquisição de imagens constituído por uma câmara digital SONY Hiper HAD, montada sobre um microscópio Olympus BX50, acoplada a um computador Pentium 200 MHZ com 128 MB de memória RAM. Preparações para o MEV foram efetuadas de acordo com a técnica de Maia \& Santos (1997). Forma e septação de conídios, espessura de hifas, forma, comprimento e espessura das estruturas de captura, ovos e nematóides predados foram documentados. Os dados obtidos foram comparados com as descrições apresentadas por McCulloch (1977).

Uma cultura do fungo isolado foi repicada para quatro placas de Petri contendo ágar-água a $2 \%$ e incubadas no escuro, à temperatura ambiente, por $48 \mathrm{~h}$. Posteriormente, para o estudo da patogenicidade do fungo, cada uma dessas placas recebeu $1 \mathrm{ml}$ de suspensão do nematóide de vida livre (NVL) Panagrellus sp. contendo, em média, 200 espécimes/ ml. Igual número de placas foi preparado com suspensão de ovos e juvenis de segundo estádio de $H$. glycines contendo, em média, 150 ovos e juvenis/placa e incubadas nas mesmas condições. Após 24 h do início da incubação, procederam-se as avaliações, tendo sido verificado que $100 \%$ das formas ativas dos juvenis e dos ovos de $H$. glycines haviam sido capturadas pelo fungo, o qual apresentava abundante esporulação. Então, as culturas foram fixadas nas placas com glutaraldeído a $3 \%$, em solução tampão

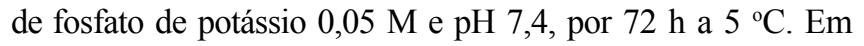
seguida, foram lavadas seis vezes consecutivas na solução tampão pura, em um intervalo de 15 min e pós-fixadas em tetróxido de ósmio a 2\%, na mesma solução tampão, por 8 $\mathrm{h}$, à mesma temperatura. Posteriormente, foram novamente lavadas, como no caso anterior. Com o auxílio de uma espátula, pequenos retângulos do meio contendo as estruturas de interesse foram retirados e transferidos para frascos de vidro. A seguir, as amostras foram desidratadas em álcool etílico, secas em secador de ponto crítico, montadas, recobertas com ouro-paládio e elétronmicrografadas em microscópio eletrônico de varredura JEOL JSM 5410, operando em 15 kV (Maia \& Santos, 1997). 


\section{RESULTADOS E DISCUSSÃO}

A Figura 1A ilustra a morfologia do conídio do fungo nematófago estudado, o qual, de acordo com as descrições apresentadas por McCulloch (1977), é típico da espécie $M$. robustum. Com efeito, os conídios fusóides, hifas mais espessas, ramos adesivos unicelulares maiores e de maior diâmetro que em outras espécies do grupo, são características marcantes da espécie. Observou-se nitidamente a presença de conídio fusóide, cuja célula mediana se mostrava saliente. Geralmente, verificavam-se de dois a quatro septos (Figura 1A), e, às vezes, foram notados até cinco septos. Os conídios maduros mediam, em média, $40 \mu \mathrm{m}$ de comprimento e 16 $\mu \mathrm{m}$ de largura $(\mathrm{n}=30)$. Na Figura 1B, foi ilustrada a presença abundante de hifas do fungo em ágar-água. As hifas apresentaram-se com 2 - $4 \mu \mathrm{m}$ de largura, em média. O crescimento das hifas tendeu a retilíneo, mas freqüentemente apareceram paralelas. $\mathrm{O}$ aspecto rugoso do micélio decorreu da penetração sistemática da hifa no meio de culturas, e retorno à superfície à semelhança de uma costura (Figuras 1B e 1C). Sobre as hifas, cresceram, verticalmente, prolon- gamentos adesivos sésseis e individuais com até $10 \mu \mathrm{m}$ de comprimento e $5 \mu \mathrm{m}$ de largura, que são as estruturas ou armadilhas de captura do fungo (Figura 1E, seta). Essas estruturas possuiam o diâmetro ligeiramente maior que o das hifas que lhes deram origem, ovóides e de aspecto mucilaginífero. Quando o nematóide entrava em contato com essa estrutura, a membrana que a recobre rompia-se, aderindo-se a uma expressiva área do corpo do nematóide, aprisionandoo (Figura 1F, seta). Como se formavam várias dessas estruturas próximas umas às outras, numa mesma hifa, ou em hifas paralelas, usualmente um nematóide foi capturado por mais de uma (Figura 1C, seta). A propriedade adesiva estava presente apenas nas estruturas de captura do fungo e não em toda a extensão da hifa. Barron (1977), utilizando-se do MEV, também observou em estudos com A. oligospora que apenas as redes tridimensionais apresentavam efeito adesivo. A presença de ovos de $H$. glycines no meio de cultura induziu intensa formação das estruturas de captura em sua proximidade (Figura 1D), muitas vezes mais numerosas do que ao redor do nematóide capturado (Figura 1C). Embora a predação de ovos por $M$. robustum tenha sido evidente

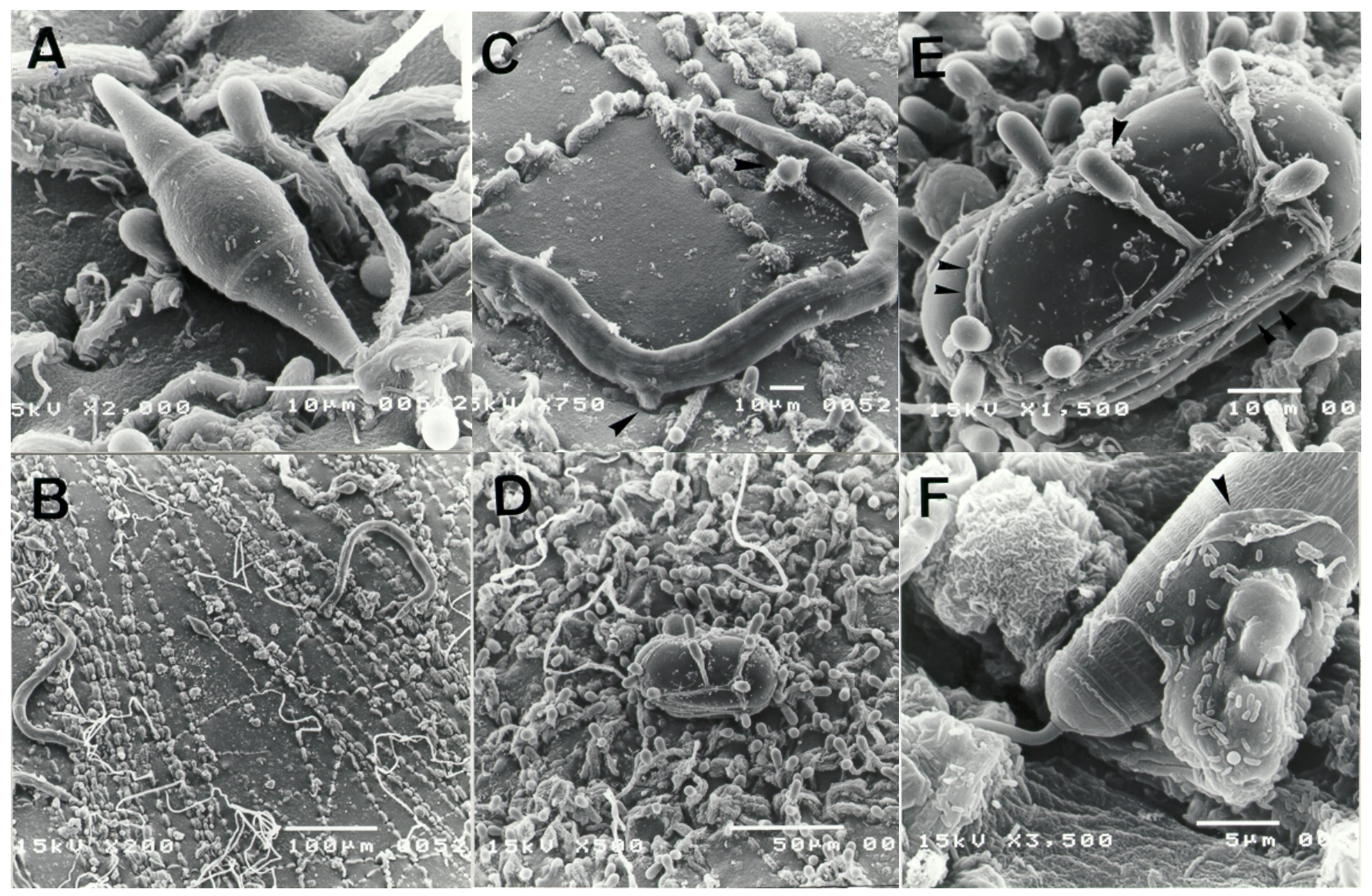

FIG. 1 - Elétron-micrografias de varredura de Monacrosporium robustum. A) Conídio; B) Aspecto da cultura em ágarágua e juvenis de segundo estádio de Heterodera glycines capturados pelo fungo; C) Detalhes da cultura do fungo e do juvenil aprisionado pelo fungo $(\uparrow)$; D) Ovo predado pelo fungo e numerosos ramos adesivos em sua proximidade; E) Ovo de Heterodera glycines predado pelo fungo exibindo ramos adesivos $(\uparrow)$ e áreas sob pressão das hifas do fungo $(\uparrow \uparrow)$; F) Aspecto de um ramo adesivo do fungo com a porção mucilaginosa $(\uparrow)$ e presença de bactérias tipo bastonete. 
(Figuras 1D e 1E), não se constatou parasitismo. Contudo, observou-se a pressão exercida pelas hifas sobre a parede externa do ovo (Figura 1E, setas). De fato, as espécies de Monacrosporium não são incluídas entre os parasitos de ovos. Mankau (1980) incluiu Monacrosporium spp. entre os fungos parasitos de nematóides vermiformes, ao lado de Arthrobotrys Corda, Dactylaria Saccardo e Dactylella Grove. Contudo, Jatala (1986) mencionou que muitos dos organismos que primariamente parasitam um certo estádio de desenvolvimento de um nematóide, podem, ocasionalmente, parasitar outros estádios. Todavia, a habilidade de determinado fungo em infectar vários estádios de desenvolvimento do nematóide, ao mesmo tempo ou com a mesma freqüência, não é comum.

Com o desenvolvimento da biologia molecular, técnicas novas irão permitir a identificação das substâncias diretamente envolvidas na interação nematóide-fungo, possibilitando o conhecimento das bases bioquímicas da interação e ampliando as possibilidades de interferência no processo, quer selecionando agentes de controle biológico com maiores chances de sucesso no controle de certos nematóides, quer interferindo no ambiente, visando-se aumentar a eficiência de um determinado sistema nematóide-fungo.

No cultivo de $M$. robustum em ágar-água a $2 \%$, no presente estudo, observou-se a presença constante de uma bactéria em forma de bastonete, ainda não identificada. Referida bactéria usualmente se encontrava associada à estrutura de captura do fungo, notadamente após a adesão ao corpo do nematóide (Figura 1E). Duponnois et al. (1998) relataram que existem bactérias associadas a fungos nematófagos, as quais podem interferir na ação de predação e parasitismo, especificidade, agressividade e reprodução dos fungos. Muitos isolados testados por esses autores aumentaram a ação do fungo. Essas bactérias foram denominadas NHB ("Nematophagous Fungus Helper Bacteria"). Estes autores relataram que Meloidogyne mayaguensis Rammal e Hirschmann, 1988 e M. incognita foram mais atacados por $A$. oligospora do que por $M$. javanica. Porém, quando os juvenis foram colocados na presença do fungo associado à bactéria, não houve especificidade, e a agressividade do fungo aumentou. A literatura especializada mostra que outras bactérias estimularam a atividade dos órgão de captura de fungos na presença de espécies de Meloidogyne, aumentando a agressividade do fungo. Citando alguns autores, Duponnois et al. (1998) aventaram a hipótese de que essas bactérias poderiam produzir exopolissacarídeos ou compostos tóxicos. Tais substâncias reduziriam a mobilidade dos juvenis ou poderiam, também, produzir oligossacarídeos ou açúcares simples, os quais atuariam como ponte molecular entre os dois microrganismos, reduzindo, também, a especificidade entre fungos e nematóides. Contudo, o papel dessas bactérias, ao certo, ainda é desconhecido. Considerando-se que a presença da bactéria observada no presente estudo, o isolado em questão de $M$. robustum, entre os vários outros fungos estudados no passado pelos autores, até o presente, foi o que exibiu maior potencial de controle biológico de $H$. glycines.

\section{REFERÊNCIAS BIBLIOGRÁFICAS}

BARRON, G.L. The nematode-destroying fungi. Guelph. Canadian Biological Publications Ltd. 1977.

CARNEIRO, R.M.D.G. \& GOMES, C.B. Metodologia e teste de patogenicidade de Paecilomyces lilacinus e $P$. fumosoroseus em ovos de Meloidogyne javanica. Nematologia Brasileira 7:66-75. 1993.

CHEN, S.Y.\& DICKSON, D.W. Fungal penetration of the cyst wall of Heterodera glycines. Biological Control 86:319-327. 1996.

CHEN, S.Y., DICKSON, D.W., KIMBROUGH, J.W., McSORLEY, R. \& MITCHELL, D.J. Fungi associated with females and cysts of Heterodera glycines in a Florida soybean field. Journal of Nematology 26:296303. 1994.

CHEN, S.Y, DICKSON, D.W. \& MITCHELL, D.J. Pathogenicity of fungi to eggs of Heterodera glycines. Journal of Nematology 28:148-158. 1996.

COIMBRA, J.L., CAMPOS, V.P. \& SOUZA, R.M. de. Isolamento e parasitismo de fungos de fêmeas de Meloidogyne javanica e Meloidogyne exigua. Nematologia Brasileira 23:25-33. 1999.

DALLA PRIA, M. Controle biológico de Meloidogyne incognita Raça 3, pelos fungos Verticilium chlamydosporium e espécies de Monacrosporium, isolados ou combinados. (Tese de Mestrado). Viçosa. Universidade Federal de Viçosa. 1992.

DUPONNOIS, R., AMADOU, M.B. \& MATEILLE, T. Effects of some rhizospher bacteria for the biocontrol of nematodes of the genus Meloidogyne with Arthrobotrys oligospora. Fundamental and Applied Nematology 2:157-163. 1998.

FERRAZ, S., MAIA, A.S., MUCHOVEJ, J.J. \& SANTOS, J.M. dos. Detecção, isolamento, identificação e avaliação "in vitro" da capacidade predatória de fungos nematófagos de solos brasileiros. Nematologia Brasileira 16:85-86. 1992.

FREITAS, L.G., FERRAZ, S. \& ALMEIDA, A.M.S. Controle de Meloidogyne javanica em tomateiro pela produção de mudas em substrato infestado com Paecilomyces. Nematologia Brasileira 23:65-73. 1999.

JATALA, P. Biological control of plant-parasitic nematodes. Annual Review of Phytopathology 24:453-489. 1986.

MAIA, A.S. \& FERRAZ, S. Detecção e isolamento de fungos nematófagos de solos brasileiros. Resumos, $17^{\circ}$ Congresso Brasileiro de Nematologia, Jaboticabal, SP. 1993. p. 68.

MAIA, A.S., FERRAZ, S. \& DALLA PRIA, M. Detecção, isolamento e identificação de Monacrosporium spp. Resumos, $17^{\circ}$ Congresso Brasileiro de Nematologia, Jaboticabal, SP. 1993. pp.69.

MAIA, A. S. \& SANTOS, J. M. dos. Microscopia eletrônica de varredura das estruturas de captura de alguns fungos nematófagos. Acta Microscopica 4:304. 1995.

MAIA, A.S. \& SANTOS, J.M. dos. A SEM technique for 
preparing biological control agents of nematodes in action. Acta Microscopica 6:550-551. 1997.

MAIA, A.S. \& SANTOS, J. M. dos. SEM study of nematophagous fungi in action. Acta Microscopica 8:615-616. 1999.

MANKAU, R. Biological control of nematode pests by natural enemies. Annual Review of Phytopathology 18:415-440. 1980.

McCULLOCH, J.S. New species of nematophagous fungi from Queensland. Transactions of the Chemical Ecology 18:775-783. 1977.

MIZOBUTSI, E.H., FERRAZ, S., RIBEIRO, R.C.F. \& MENEZES, M. Isolamento de fungos de ovos de Heterodera glycines coletados em diferentes regiões produtoras de soja do Brasil. Nematologia Brasileira 23:.69-75. 1999.

NAVES, R.L. \& CAMPOS, V.P. Ocorrência de fungos predadores de nematóides no Sul de Minas Gerais e estudos da capacidade predatória e crescimento in vitro de alguns de seus isolados. Nematologia Brasileira 15:152-162. 1991.

PERSSON, C. \& JANSSON, H-B. Rhizosphere colonization and control of Meloidogyne spp. by nematode-trapping fungi. Journal of Nematology 31:164-171. 1999.

RIBEIRO, R.C.F., FERRAZ, S. \& MIZOBUTSI, E.H. Avaliação da eficiência de isolados de Monacrosporium spp. no controle de Meloidogyne javanica e Heterodera glycines. Nematologia Brasileira 23:48-61. 1999a.

RIBEIRO, R.C.F., FERRAZ, S., MIZOBUTSI, E.H. \& MENEZES, M. Levantamento de espécies de Monacrosporium predadoras de nematóides de diversas regiões brasileiras. Nematologia Brasileira 23:41-47. $1999 b$.

SANTOS, M.A. Detecção, identificação e avaliação do potencial antagonista de fungos nematófagos em solos do Brasil (Tese de Mestrado). Viçosa. Universidade Federal de Viçosa. 1991.

SANTOS, M.A. Estudo de alguns fungos endoparasitos e predadores no controle de fitonematóides (Tese de Doutorado). Viçosa. Universidade Federal de Viçosa. 1996.

SANTOS, M.A. dos, FERRAZ, S. \& MICHOVEJ, J.J. Detection and ecology of nematophagous fungi from Brazilian soils. Nematologia Brasileira 15:121-134. 1991. 